\title{
Comparative analysis of human development indicators: Tanger-Tetouan-Al Hoceima region
}

\author{
Ikrame Abroun ${ }^{1}$, Abdelilah Azyat ${ }^{2}$, Nizar Ben Achhab ${ }^{1}$, Naoufal Raissouni ${ }^{1}$, and Asaad \\ Chahboun ${ }^{1}$ \\ ${ }^{1}$ RSGIS Lab. Remote Sensing GIS Unit, ENSA of Tetouan, Morocco \\ ${ }^{2}$ MIA Research Unit, ENSA of Tangier, Morocco
}

\begin{abstract}
Human development is more than a question of the accumulation of wealth, income, or economic growth. It must be human-centred. This is why concerns as necessary as respect for human rights, the reduction of social inequalities and poverty, the promotion of equal opportunities between men and women are indeed relevant. This considers human resources not only as a means of growth but, more fundamentally, as an end of growth. The demographic variable was always a serious problem to decision-makers in different countries. It is considered to be at the root of the various handicaps of development. Morocco has carried out throughout the last forty years' population policies to improve the well-being of its citizens. To highlight regional and provincial disparities in Morocco, we are based in this work on indices of human development, namely, SDI, ASDI, MLDI using the process of the data warehouse. Finally, we have analyzed and visualized these indices with Power BI software to make a comparative analysis of TTA provinces. Findings show that Tanger-Assilah province has a great value with 0.77 of MLDI. However, Chefchaouen province has the less one with 0.56 . This study has been performed to help to establish efficient decisions and making operational insights.
\end{abstract}

\section{Introduction}

With the publishing of its first annual Human Development Report in 1990 (HDR), the United Nations Development Program (UNDP) changed the perception of development theory, measurement, policy, and the introduction of the Human Development Index. HDR 1990 presented the concept of "human development" as progress towards greater human wellbeing, and provided country-level data for a wide range of wellbeing indicators. The UNDP's establishment of the HDR expanded both the availability of measurement and comparison tools used by governments, NGOs, and researchers and our common understanding of development itself. Key capabilities are instrumentals in HDI including proxies for three important ends of development: access to health, education, and goods [1]. While the HDI allows for a comparison ranking of countries, it has flaws, such as not being highly descriptive at finer geographical scales. It also lacks a dynamic and less static explanation of observed progress or delays. To address these limitations, we will use indicators that are more adapted to the Moroccan scenario, are available at sub-national scales, and incorporate two elements of the HDI, namely education and health. We aim to 
diagnose and resolve a challenge in our work: how to measure the degree of human and social development at the provincial level while accounting for regional variations, and how to determine inter-regional disparities and therefore uncover continuous deficits [2].

\section{Human development indices}

\subsection{Human development index HDI}

The HDI is an index that evaluates various aspects of human development. The three main dimensions are: a long and healthy life measured by life expectancy expressed as Ih, access to education measured by expected years of schooling of children at school-entry age and mean years of schooling of the adult population described as Ie, and a decent standard of living measured by Gross National Income (GNI) per capita adjusted for the price level of the country described by Iin [3].

After each of the component indices has been defined, the HDI is calculated by combining them. As shown in (1), the HDI is determined as the geometric mean of life expectancy, education, and GNI per capita:

$$
H D I=(I h * I e * \operatorname{Iin})^{\frac{1}{3}}
$$

\subsection{Social development index SDI}

It is a two-dimensional indicator proposed by the [3] National Observatory for Human Development and based on education and health. The rate of medical supervision measured at the provincial level is employed as an indicator for the health dimension denoted by the medical index MI. The success rate at the end of the college cycle is used as the indicator for the education dimension, which is stated in the school index SI.

The proposed SDI is defined as the arithmetic mean of the SI and MI indices computed for each of the two dimensions (2).

The higher the SDI, the higher the level of human development in a specific area [3]. Its value might range from 80 to more than 125 points.

$$
S D I=\frac{S I+M I}{2}
$$

\subsection{African social development index ASDI}

This index, also known as the human exclusion index, was proposed by High Commission for Planning (HCP) and developed in collaboration with the United Nations Economic Commission for Africa (ECA). It allows for the diagnosis of socio-economic development at the national and local levels in order to measure the impact of public resources on the populations who need them the most [4].

The dimensions thus retained for its calculation relate to infant mortality expressed by $\mathrm{dEx}^{\mathrm{im}}$, child malnutrition defined by $\mathrm{dEx}^{\mathrm{chm}}$, illiteracy defined by $\mathrm{dEx}^{\mathrm{Lr}}$ and unemployment among young people aged 15-24 expressed by $\mathrm{dEx}^{\mathrm{Yu}}$,poverty measured at the national 
threshold expressed by $\mathrm{dEx}^{\mathrm{np}}$ and finally life expectancy at 60 years expressed by $\mathrm{dEx} \mathrm{x}^{\mathrm{Le}}$ [2].We aggregate the levels of exclusion in each of the six dimensions [4] as showed in (3):

$$
A S D I=\underset{d E x^{L e}}{d E x^{i m}}+d E x^{c h m}+d E x^{L r}+d E x^{Y u}+d E x^{n p}+
$$

ASDI's value can range between 0 and 1 , and it varies in the opposite direction, i.e. The lower the ASDI, the less the region is affected by human exclusion.

\subsection{Multidimensional local development index MLDI}

The MLDI is a measure of development based on indicators available at the municipal level [4].It is based on six dimensions are: the health dimension which is measured by the chances of survival beyond 70 and life expectancy at 60 and expressed by HI, education by the literacy rate, the proportion of the population knowing Arabic and French and the proportion of the population having completed college, high school or higher expressed by EI, employment by unemployment rate and paid employment measured by EmI, the housing condition by the percentage of households living in non-basic housing, villas or Moroccan houses, etc and which is expressed by ShI, access to social service by SsI, and finally, the living environment expressed by LeI.

The dimensional indices are calculated for each of the six dimensions selected. Their simple arithmetic mean gives the MLDI value. Its value varies between 0 and 1 [4]:

$$
M L D I=\sum_{1}^{6} \frac{H I+E I+E m I+S h I+S S I+L e I}{6}
$$

\section{Methodology}

\subsection{Decision-making}

Decision making is to assess and make choices. This decision was taken after going through several calculations and alternative considerations. Before the choice is made, there are several stages that may be passed by the decision maker. This stage may include identifying the main problems, developing alternatives to be chosen and arriving at the best decision making [5]. Infographics, charts, plots, graphs, and maps play an important role in decisionmaking because they synthesize and display the findings [6].

\subsection{Data collection}

Data collection is a systematic process of gathering observations or measurements, it allows you to gain first-hand knowledge and original insights into your research problem. Whatever philosophical standpoint the researcher is taking and whatever the data collection method the process will involve the generation of large amounts of data [7]. 


\subsubsection{Data sources}

The data used in this project are those of the three indices SDI, MLDI and ASDI for TTA region in 2014. We obtained this data from ONDH on which we have based our calculations for SDI and also another data obtained from HCP which concerns the index ASDI [8].

The index calculated in this project is the SDI which is expressed as the arithmetic mean of the indicators obtained for each of the two dimensions, education and health, as mentioned. The higher the SDI, the more satisfactory the level of human development of the territory is [8].

Remark: Because of the lack in data, we had to work with previously calculated indexes such as ASDI and MLDI [8] .

\subsection{Data Modelling}

Data modelling is the process of creating a data model for the data to be stored in a database. This data model is a conceptual representation of data objects, the associations between different data objects, and the rules [9].Star schema is the most common modeling paradigm [10].

\subsubsection{Data warehouse}

A data warehouse is a database designed for the business intelligence requirements and managerial decision making of an organization. The data warehouse integrates data from the various operational systems and is typically loaded from these systems at regular intervals. It contains historical information that enables the analysis of business performance over time. The data are subject oriented, integrated, time variant, and nonvolatile [11]. For this reason, there are several challenges associated with building data warehouses, these challenges are data quality, understanding data, testing, performance, designing the DW, cost, and security [12].

Basically, external data source major step involved in integration of data to data warehouse as discussed above which are four steps [13]: Data source identification, data extraction, data transformation and data loading.

\subsection{Data storage}

Data storage is a general term for archiving data in electromagnetic or other forms for use by a computer or device.

\subsection{Database visualization}

In order to analyze our data, we have visualized them used Power BI software which is a cloud-based business analytics service from Microsoft that enables anyone to visualize and analyze data, with better speed and efficiency. It is a powerful as well as a flexible tool for connecting with and analyzing a wide variety of data. This feature helps to perform tasks like sorting, comparing and analyzing, very easily and fast. First, we visualized the data obtained by ONDH of each prefecture of TTA region in 2014.We follow the level of social development in each province by analyzing SDI. Secondly we analyzed the MLDI. Then, we compared the results obtained by the analysis of the SDI and the visualization results of the 
MLDI in order to assess and identify social deficits to measure the impact of public resources on the populations who need them the most. And then, we will perform the visualizations of ASDI.

\section{Results}

\subsection{Data analysis}

\subsubsection{School delay for the TTA region}

The delay in the college cycle varies from one province to another in TTA region. This variation fluctuates between 1.17 in M'diq-Fnidaq and 1.92 in Chefchaouen. So a child from Chefchaouen province can easily fall behind by one school year more than a child from M'diq-Fnidaq or Tetouan compared to the normal age. Compared to the national average, only the provinces of M'diq-Fnidaq-Tetouan and the prefecture of Tangier-Assilah which are in a comfortable situation. The other five provinces have significantly higher delays.

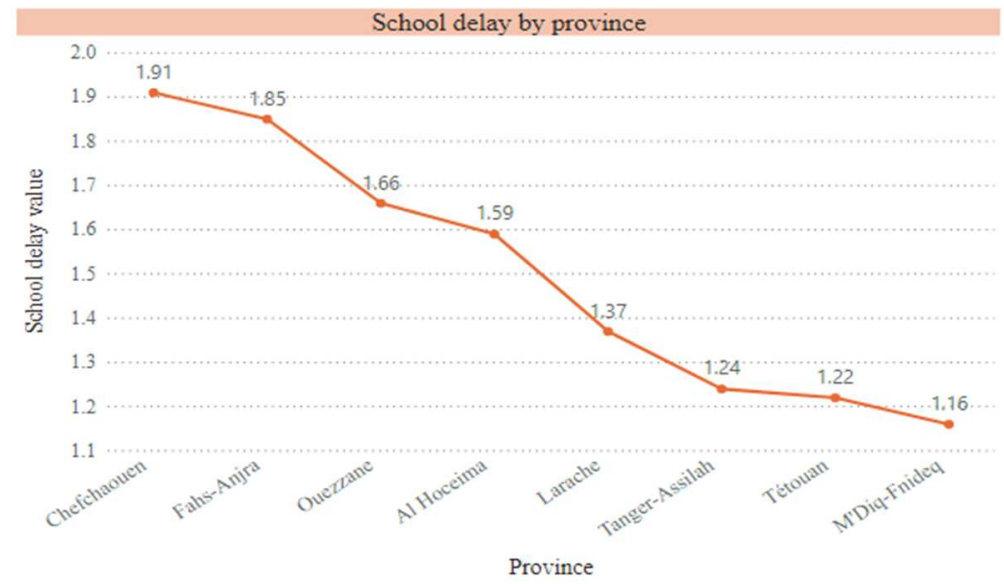

Fig.1. School delay for the TTA region

\subsubsection{Public medical supervision in the TTA region}

For the TTA region, only the provinces of Al Hoceima, Tetouan and that of M'diq-Fnidaq, which have a satisfactory average compared to the national average in terms of medical supervision. While for the other provinces of the region, interventions must be carried out to compensate for the denoted deficit. 


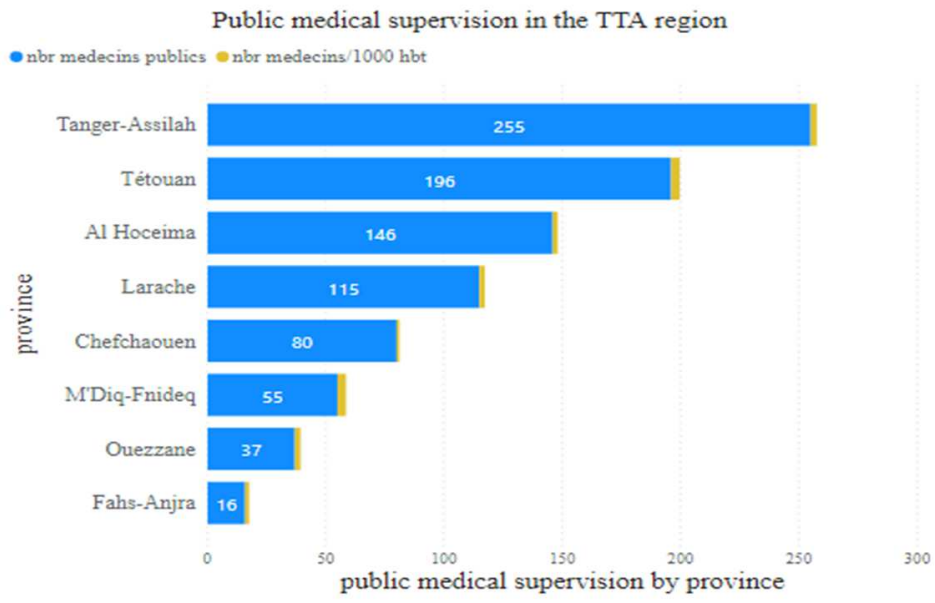

Fig. 2. Public medical supervision in the TTA region

\subsubsection{School and medical index for the TTA region}

In the case of the provinces of Tangier-Tetouan-al Hoceima region, there are overall deficits in education and health, more particularly for the province of Chefchaouen, followed by that of Ouezzane which is moderately good in terms of its school index. In the average performance zone, we find the prefecture of Tangier-Assilah and the province of Larache, which are characterized by acceptable levels of performance in both sectors. The province of Fahs-Anjra is at an average level in health but requires efforts in the education sector. The province of Al Hoceima is in a comfortable health situation but clearly out of step with regard to education. The two provinces which note satisfactory performances in education and health are the provinces of M'diq Fnidaq and Tetouan.

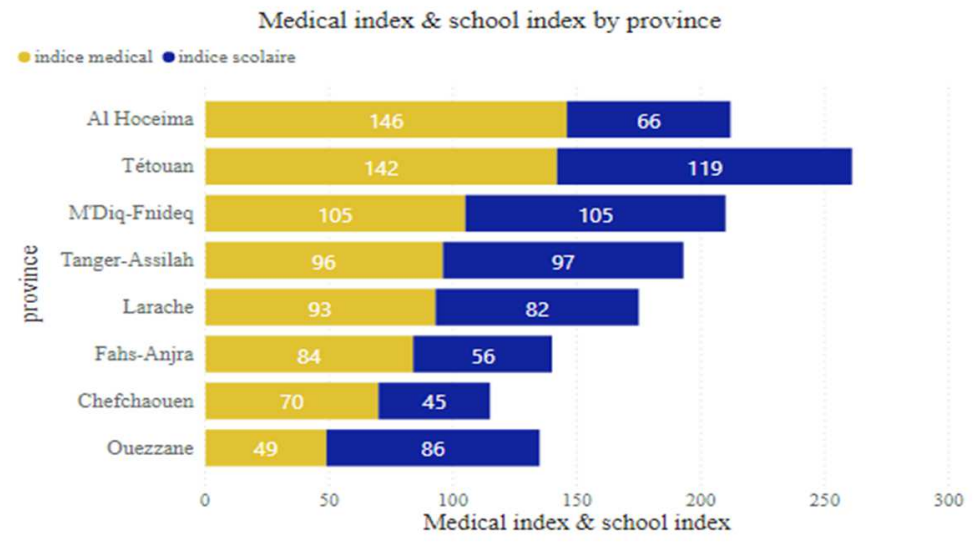

Fig. 3. School and medical index for the TTA region 


\subsubsection{Social development index SDI for the TTA region}

Based on the arithmetic mean of the two indices SI and MI, we obtain as a product an index which synthesizes the level of social development in each of the prefectures/ provinces of the region.

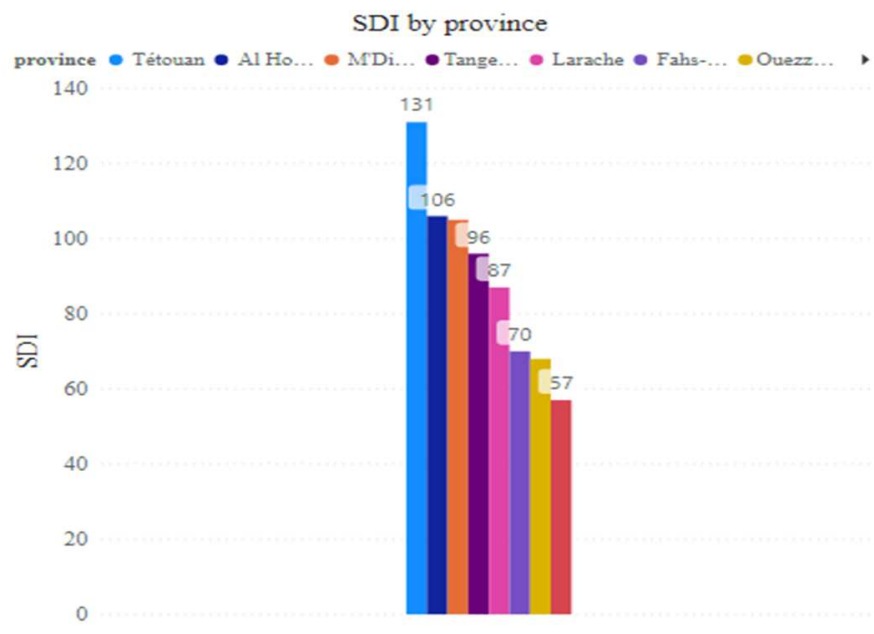

Fig. 4. SDI for the TTA region

\subsubsection{ASDI by region}

At the regional level, human exclusion is more frequent in the regions of Fès-Meknes 1.05, the regions of the south 1.0 and Souss-Massa 1.0. The human exclusion index is close to that of the national average in the regions of Béni-Mellal-Khénifra 0.96, Marrakech-Safi 0.98, and Darâa-Tafilalet 0.98. The regions least affected by human exclusion are CasablancaSettat 0.67, Rabat-Salé-Kénitra 0.83 Oriental 0.74 and Tanger-Tetouan-Al Hoceima 0.85.

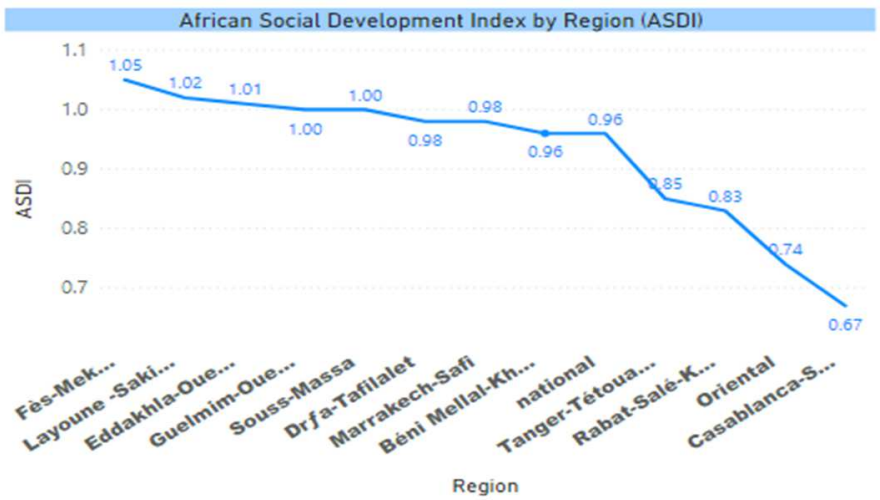

Fig. 5. ASDI by region

\subsubsection{Multidimensional local development index MLDI for the TTA region}

The mountainous regions, manly rural and agricultural, where the lowest levels of development were recorded. These are primarily Chefchaouen 0.555, Ouezzane 0.606, 
followed by Fahs-Anjra 0.612, and Al Hoceima 0.613. The most developed provinces are Tanger-Assilah 0.770 , and M'diq-Fnidaq 0.757 . The rest of the provinces, namely, Tetouan and Larache, with values respectively 0.716 and 0.662 are those whose's level of development is relatively closer to the national average.

MLDI by province

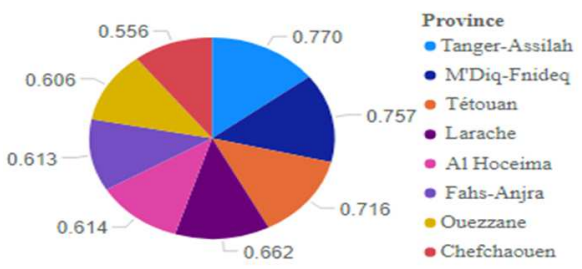

Fig. 6. MLDI by province

\subsubsection{Comparison of SDI, MDLI and ASDI for TTA region}

The results of this analysis made it possible to pin point the best performing provinces, both in terms of education and health, and subsequently to deduce their situations in terms of social development. Indeed, for the TTA region, and based on the results of the SDI. The region obtains an SDI aligned with the national average with a value of 93.

The index also made it possible to identify the provinces which are at the top and bottom of the list. M'diq Fnidaq 105, Al Hoceima 106 and Tetouan 131, are in the zone of good performance in education and health. The prefecture of Tangier-Assilah 96 and the province of Larache 87 are both in the average performance zone, with acceptable levels of performance in both sectors. The province of Fahs-Anjra 70 has an average level of health, but education needs improvement. The provinces of Chefchaouen 57, followed by that of Ouezzane 68 are the most affected and therefore need rapid interventions in the fields of health and education.

Regarding the MLDI, it shows that TTA among regions whose level of development is significantly lower than the national average of the MLDI 0.700 with a value of 0.683 . The underdevelopment of the region is attributed, according to the results of MLDI, to its mountainous areas, difficult to access and under-equipped in social infrastructure. The most developed provinces are Tanger-Assilah 0.770, and M'diq-Fnidaq 0.757. Tetouan 0.716 and Larache 0.662 have a level of development comparable to the national average. And finally, the lowest levels of development were recorded in Chefchaouen 0.555, Ouezzane 0.606, Fahs-Anjra 0.612, and Al Hoceima 0. 613.The two indices SDI and MLDI give almost the same results concerning the order of the provinces at the level of their social development. In fact, we find Chefchaouen, Ouezzane and Fahs-Anjra are still among the most affected prefectures. Unlike Tanger-Assilah, M'diq-Fnidaq, Tetouan and Larache vary between the two good and medium performance classes.

The ASDI index has values only on the regional level; it shows that TTA region belong to the regions least affected by human exclusion with an ASDI value equal to 0.85 (Fig.5). 
Table 1 . Comparison of SDI, MLDI and ASDI

\begin{tabular}{|l|c|c|}
\hline \multirow{2}{*}{ Province } & \multicolumn{2}{|c|}{ Indices } \\
\cline { 2 - 3 } & SDI & MLDI \\
\hline Tetouan & 131 & 0.716 \\
\hline M'Diq-Fnideq & 105 & 0.757 \\
\hline $\begin{array}{l}\text { Tanger- } \\
\text { Assilah }\end{array}$ & 96 & 0.77 \\
\hline Ouezzane & 68 & 0.606 \\
\hline $\begin{array}{l}\text { Larache } \\
\text { Al Hoceima }\end{array}$ & 106 & 0.614 \\
\hline $\begin{array}{l}\text { Fahs-Anjra } \\
\text { Chefchaouen }\end{array}$ & 70 & 0.613 \\
\hline National & 93 & 0.556 \\
\hline
\end{tabular}

\section{Conclusion}

The work presented in this article has as a goal analyzing and comparison of the human development indicators according to regions and at most delicate territorial scales. The analysis carried out in this context makes it possible to identify the provinces that show the most disparities in terms of social development. This analysis was essentially based on the modelling of a decision-making system that serves, via a multitude of functionalities it offers, decision-making assistance and advocacy tool for the detection of corridors of territorial deficits in education and health in the eight provinces of the TTA region. Therefore, future prospects are to carry out a thorough and precise study for the construction of a strategic plan based on a geographic information system (GIS), taking into account the indicators mentioned to measure sustainable development in the TTA provinces.

The authors would like to thank the reviewers and anonymous referees for providing helpful comments and suggestions which led to an improvement of the paper. 


\section{References}

[1] E. A. Stanton, «The human development index: A history,» PERI Working Papers, (2007).

[2] I. Abroun,A.Azyat «Analysis and diagnosis of data to determine indicators and developement issues for the Tanger-Tetouan-Al Hoceima region,» Tangier, Morocco, (2020).

[3] M. Roser, «Human Development Index (HDI),» Our World in Data, (2014).

[4] «Le développement socio-économique régional ,Niveau et disparités, 2001-2017» HCP, Morocco, (2017).

[5] «African social development index (asdi): measuring human exclusion for structural transformation,» 68, pp. 1-78, (2016).

[6] «Cartographie du développement local multidimensionnel niveau et déficit,» ONDH.

[7] Meiryani, P.Siagian,RA.Aryanti Wardaya Puspokusumo, Lusianah, «DECISION MAKING AND MANAGEMENT INFORMATION SYSTEMS,» JCR, 7, pp. 320-325, (2020).

[8] Alam, Furqan,Almaghthawi, Ahmed and Katib, Iyad and Albeshri, Aiiad and Mehmood, Rashid, «iResponse: An AI and IoT-Enabled Framework for Autonomous COVID-19 Pandemic Management,» Sustainability, 13, (2021).

[9] J.Sutton, \& Z. Austin, «Qualitative Research: Data Collection, Analysis, and Management.,» Can. J. Hosp. Pharm., 268,3, p. 226-231, (2015).

[10] A. Azyat. N. BenAchhab, N. Raissouni , A. Chahboun. I.Abroun, «Mapping human developement indices in Moroccan regions,» p. 7, (2021).

[11] Mazumdar, Somnath and Seybold, Daniel and Kritikos, Kyriakos and Verginadis, Yianni, «A survey on data storage and placement methodologies for cloud-big data ecosystem,» Journal of Big Data, 6, pp. 1--37, (2019).

[12] Pei, Jiawei Han and Micheline Kamber and Jian, Data Mining (Third Edition), Third Edition éd., J. H. a. M. K. a. J. Pei, Éd., Boston: Morgan Kaufmann, (2012).

[13] Shahri, H.Haidarian, A Machine Learning Approach to Data Cleaning in Databases and Data Warehouses, University of Maryland, USA, (2008), p. 15.

[14] Shen, M.AlMeghari and S.Taha and H.Elmahdy and X.(Sherman), «A proposed authentication and group-key distribution model for data warehouse signature, DWS framework,» Egyptian Informatics Journal, (2020).

[15] Wah, T.Ying and Peng, N.Hooi and H. Ching Sue, «Building data warehouse,» chez Proceedings of the 24th South East Asia Regional Computer Conference, 15, (2007), pp. 51--56. 